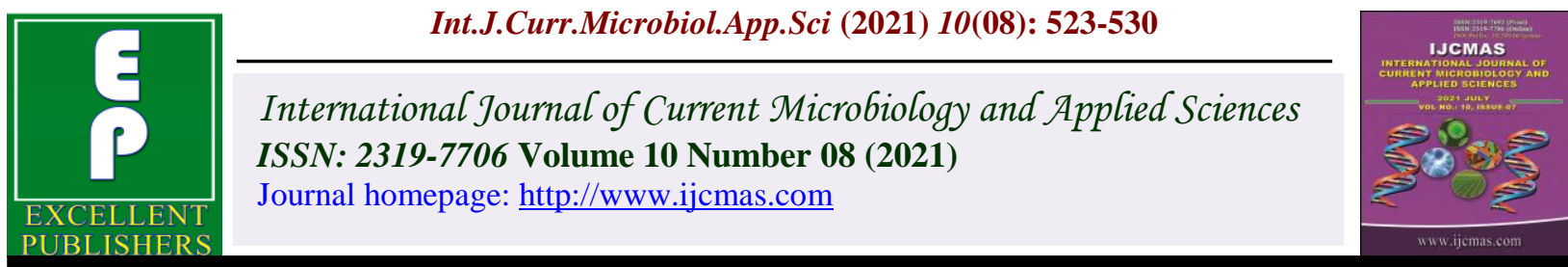

\title{
Coagulase-Negative Staphylococci (CoNS) Positivity and Sensitivity in Invasive Blood Stream Infections using Automated Bactec in Tertiary care Hospital of a Hilly State in India
}

\author{
Pankaj Katoch $^{1 *}$, Ambika Sood ${ }^{2}$ and Surinder Singh ${ }^{2}$ \\ ${ }^{1}$ Department of Microbiology, ${ }^{2}$ Department of Paediatrics, IGMC, Shimla, India \\ *Corresponding author
}

A B S T R A C T

\section{Keywords}

Blood Stream Infections, Culture, Bactec, Positivity, Sensitivity, Coagulase-Negative Staphylococci (CoNS)

Article Info

Accepted: 20 July 2021 Available Online: 10 August 2021
Today, in the present-day scenario CoNS, as typical opportunistic infections, represent one of the significant nosocomial pathogens, having a huge impact on human health and life. It is very difficult to determine whether positivity is due to bacteremia or contamination. Hence, the present study is done to determine the Coagulase-Negative Staphylococci (CoNS) positivity in Invasive bloodstream infections using Automated Bactec systems. All the blood culture samples received in the Department of Microbiology for culture by Bactec Bd fx from July 2015 to June 2016 were included in the study. The blood culture was observed in the Bactec Bd fx system for at least 5 days before they are reported as sterile. Among the total 1275 cultures which were positive for bacteria, 344(26.98\%) were of Gram-positive bacteria while 931(73.02\%) were positive for Gram-negative bacteria. Among the total of 344 culture positive for Gram-positive bacteria, CoNS was isolated in 143 (41.57\%) cultures. Among the total, maximum CoNS were found in the age group of 0-1 years (35.66\%) followed by the 19-45 year age group (20.98\%). All the Coagulase-negative Staphylococcus (CoNS) isolates showed $100 \%$ sensitivity to Vancomycin, Gentamycin, Teicoplanin, and Linezolid. Besides, that $91.67 \%$ of isolates were sensitive to Levofloxacin, $87.5 \%$ Tetracycline, $80 \%$ Aztreonam, and $72 \%$ to Piperacillin. It was found that Coagulasenegative Staphylococcus (CoNS) showed high-level resistance to Penicillin (53.85\%), Ceftazidime $(52.00 \%)$ Clindamycin $(47.01 \%)$, and Cotrimoxazole (41.96\%). CoNS was one of the most common organisms among GPC isolates and the commonest isolated in the neonate and infant age group. All the CoNS isolates showed maximum sensitivity to Vancomycin, Gentamycin, Teicoplanin, and Linezolid while it showed high-level resistance to Penicillin, Ceftazidime, Clindamycin, and Cotrimoxazole. Strict aseptic procedures to be implemented during patient's stay in the hospital to minimize nosocomial infections. 


\section{Introduction}

Coagulase-negative staphylococci (CoNS) are a group of pathogens that are increasingly associated with significant nosocomial infection and are one of the leading causes of bloodstream infection in patients. (BSI). ${ }^{1}$

Coagulase-negative staphylococci (CoNS) are a type of staphylococcus bacteria that commonly reside on a person's skin.

Physicians and Surgeons typically consider CoNS bacteria as relatively harmless when it remains outside the body. However, the bacteria can cause serious infections when present in large numbers, or when present in the bloodstream. ${ }^{2}$

A dozen of the over 50 described CoNS species have been commonly reported, including $S$. epidermidis, S. haemolyticus, $S$. lugdunensis, $S$. warneri, $S$. xylosus, $S$. hominis, S. capitis, S. simulans, S.sciuri, S. cohnii, S.lentus, and S. chromogenes, although other rare species, such as S.pettenkoferi, have been documented. ${ }^{3}$

As pathogens, CoNS can be involved in diverse types of diseases in deep-seated organs, including bones, the central nervous system, the heart, or joints.

They cause prosthetic valve endocarditis, focal lesions, urinary tract infections, central venous devices, and septicemia are reported, However, CoNS are typical opportunistic bacteria that can not only colonize healthy individuals but also represent one of the major nosocomial pathogens with a substantial increasing impact on human health and life. The presence of CoNS in the skin and mucous membranes of the host is the main source of endogenous infections. In addition, bacteria are transmitted among diverse hosts by crossing species barriers and even during medical procedures, especially invasive ones. ${ }^{3}$ According to a review, most CoNS infections are generally nosocomial. This means a person is exposed to the bacteria in a hospital environment. A person may have had surgery or an illness that required a stay in the hospital where CoNS bacteria from outside the body invade the body. ${ }^{2,3}$

Blood cultures are one of the most important and frequently performed tests in the clinical microbiology laboratory to diagnose invasive bloodstream infections. ${ }^{4,5}$ Indira Gandhi Medical College(IGMC) Shimla is a tertiary care hospital and patients with above mentioned clinical conditions are treated both in the outpatient department(OPD)and inpatient department (IPD) and usually require blood culture to establish the etiological diagnosis.

Previously no study has been done in IGMC Shimla using an automated Bactec BD FX machine for blood culture. Hence, the present study is done to determine the CoagulaseNegative Staphylococci (CoNS) isolates positivity in Invasive bloodstream infections using automated Bactec systems.

The main aim and objectives of this study includes to determine the Coagulase-Negative Staphylococci (CoNS) positivity and sensitivity in Invasive bloodstream infections using automated Bactec systems.

\section{Materials and Methods}

\section{Study Design}

Prospective observational study

\section{Study Setting}

Department of Microbiology, Indira Gandhi Medical College And Hospital, Shimla 


\section{Study Period}

One year from July 2015 to June 2016

\section{Inclusion criteria}

All the blood culture samples received in the department of microbiology for blood culture by Bactec Bd fx system.

Patients willing for study.

Blood cultures from all age groups

\section{Exclusion criteria}

Patient not willing to study.

Blood cultures showing mixed growth

All the blood culture samples received in the department of microbiology for culture by Bactec Bd fx were included in the study. The blood culture is observed in the bactec bd fx system for at least 5 days before they are reported as sterile.

The sample to be tested is inoculated into the Bactec ${ }^{\text {tm }}$ plus aerobic/f culture bottle for adults and Bactec ${ }^{\mathrm{tm}}$ Peds plus/f for children which is then inserted into the $\mathrm{Bd}$ bactec fluorescent series instrument for incubation. Each bottle has a sensor that can detect the increase in co2 produced by the growth of microorganisms. The sensor monitors every 10 minutes for an increase in its fluorescence, which is proportional to the amount of co2 present. A positive reading indicates the presumptive presence of viable microorganisms in the bottle. The positive bottle is to be subcultured on Blood agar and Mac Conkey's agar plates. Following the subculture on solid media from each positive bottle a smear was prepared for gram staining from that blood culture bottle. The Gramstained smear was examined for the presence of microorganisms and a presumptive report was conveyed to respective departments. The Bood agar and Mac Conkey agar plates were incubated aerobically at $37^{\circ} \mathrm{c}$ for 24 to $48 \mathrm{hrs}$ and then observed for the growth of bacteria. All bacterial isolates were identified using standard biochemical identification methods. Staphylococcus epidermidis ATCC 12228 was inoculated along with the test organisms. All the positive isolates were stocked.

\section{Statistical analysis}

The data was analyzed using statistical analysis-epi info7. The data collected was entered into a spreadsheet. The data was checked for any missing values and completed. analysis in terms of demographic variables, positivity in the processed samples, type of species prevalent, was done using statistical software epi-info version 7(7.1.1.0).

\section{Results and Discussion}

In the present study, among the total of 5473 samples suspected of BSI's received in the Department of Microbiology, IGMC, Shimla 1441 were positive. Among the total positive culture, $1275(88.48 \%)$ were positive for Bacteria while 166 (11.52\%) were positive for Fungi. Among the total 1275 cultures which were positive for bacteria, $344(26.98 \%)$ were of Gram-positive bacteria while 931 (73.02\%) were positive for Gram-negative bacteria.

Among the total of 344 culture positive for Gram-positive bacteria, Coagulase-Negative Staphylococci (CoNS) was isolated in 143 $(41.57 \%)$ cultures. Maximum CoNS were found in age group of $0-1$ years $(35.66 \%)$ followed by 19-45 year (20.98\%), 46-65 years (19.58\%), 6-18 years $(10.49 \%),>66$ years $(7.69 \%)$ and $5.59 \%$ in $2-5$ years.(Table-1)

All the Coagulase-negative Staphylococcus (CoNS) isolates showed $100 \%$ sensitivity to 
Vancomycin, Gentamycin, Teicoplanin, and Linezolid. Besides that $91.67 \%$ of isolates were sensitive to Levofloxacin, $87.5 \%$ Tetracycline, $\quad 80 \%$ Aztreonam, $72 \%$ Piperacillin, 69.93\% Ciprofloxacin, 64\% Azithromycin, 62.94\% Erythromycin, 60\% Amikacin, 58.04\% Cotrimoxazole, 52.24\% Clindamycin and 52\% to Amoxiclav (Table-2) (Figure-2).

It was found that Coagulase-negative Staphylococcus (CoNS) showed high-level resistance to Penicillin (53.85\%), Ceftazidime (52.00\%), Clindamycin (47.01\%), Cotrimoxazole $\quad(41.96 \%) \quad$ Erythromycin (37.06\%), and Ciprofloxacin (30.07\%). (Table-2) (Figure-3).

Coagulase-Negative Staphylococci (CoNS) now represent one of the significant nosocomial pathogens, with $S$. epidermidis and $S$. haemolyticus being the most encountered species. The infection is generally seen in foreign body-related infections and infections in preterm newborns. While $S$. saprophyticus has been associated with acute urethritis, S. lugdunensis has a unique status, in some aspects resembling $\mathrm{S}$. aureus in causing infectious endocarditis especially in immunocompromised patients. ${ }^{6}$

Therapeutically, CoNS are challenging due to the large proportion of methicillin-resistant strains and increasing numbers of isolates with less susceptibility to glycopeptides. ${ }^{6}$ This can create trouble for the doctors as Cons can cause a spectrum of diseases from skin infections to endocarditis, to prosthetic joints or vascular graft diseases. Prescription of high antibiotics can cause resistance to emerge.

In the current study, among the total of 344 culture positive for gram-positive bacteria, Coagulase-Negative Staphylococci (CoNS) was isolated in $143(41.57 \%)$ cultures. Maximum CoNS were found in age group of $0-1$ years $(35.66 \%)$ followed by $19-45$ year $(20.98 \%), \quad 46-65$ years $(19.58 \%), 6-18$ years $(10.49 \%),>66$ years $(7.69 \%)$ and $5.59 \%$ in 2-5 years. All the Coagulase-negative Staphylococcus (CoNS) isolates showed 100\% sensitivity to Vancomycin, gentamycin, Teicoplanin, and Linezolid. Besides that $91.67 \%$ of isolates were sensitive to Levofloxacin, $87.5 \%$ Tetracycline, $80 \%$ Aztreonam, $72 \%$ Piperacillin, $69.93 \%$ Ciprofloxacin, 64\% Azithromycin, 62.94\% Erythromycin, $60 \%$ Amikacin, $58.04 \%$ Cotrimoxazole, 52.24\% Clindamycin and 52\% to Amoxiclav. It was found that Coagulasenegative Staphylococcus (CoNS) showed high-level resistance to Penicillin (53.85\%), Ceftazidime $\quad(52.00 \%), \quad$ Clindamycin (47.01\%), Cotrimoxazole (41.96\%) Erythromycin (37.06\%), and Ciprofloxacin $(30.07 \%)$.

Treating CoNS infections are traditionally difficult because many bacterial strains have become resistant to antibiotics. The medications doctors normally prescribe to kill the bacteria aren't effective. If a person has a CoNS infection, a doctor may perform what's called culture sensitivity tests. They'll take a sample of blood, tissue, body fluid, or all of these and send it to a laboratory. Laboratory staff will then identify the infecting organism. ${ }^{2,6}$

Doctors generally often prescribe empirically high antibiotic vancomycin to treat CoNS infections. They usually give this medication through the Intravenous route but it has nephrotoxicity as a side effect. A characteristic feature of CoNS is the presence of biofilms and persister cells in central venous lines infections or vascular grafts infection. 
Table.1 Age group Distribution of CONS infection

\begin{tabular}{|c|c|c|c|}
\hline S.No. & AGE-GROUP & Frequency & Percent \\
\hline $\mathbf{1 .}$ & $0-1$ & 51 & $35.66 \%$ \\
\hline $\mathbf{2 .}$ & $2-5$ & 8 & $5.59 \%$ \\
\hline $\mathbf{3 .}$ & $6-18$ & 15 & $10.49 \%$ \\
\hline $\mathbf{4 .}$ & $19-45$ & 30 & $20.98 \%$ \\
\hline $\mathbf{5 .}$ & $46-65$ & 28 & $19.58 \%$ \\
\hline $\mathbf{6 .}$ & $\geq 66$ & 11 & $7.69 \%$ \\
\hline $\mathbf{7 .}$ & Total & 143 & $100 \%$ \\
\hline
\end{tabular}

$(n=143)$

Table.2 Sensitivity and Resistance of CoNS

\begin{tabular}{|c|c|c|c|c|c|c|c|}
\hline Drugs & Sensitive & ses\% & Resistance & res\% & Intermediate & inter\% & Total \\
\hline Amika & 15 & 60.00 & 10 & 40.00 & 0 & 0.00 & 25 \\
\hline Amoxiclav & 13 & 52.00 & 12 & 48.00 & 0 & 0.00 & 25 \\
\hline Ampicill & 66 & 46.15 & 77 & 53.85 & 0 & 0.00 & 143 \\
\hline Ampisal & 12 & 48.00 & 13 & 52.00 & 0 & 0.00 & 25 \\
\hline Azithromy & 16 & 64.00 & 9 & 36.00 & 0 & 0.00 & 25 \\
\hline Aztreon & 20 & 80.00 & 5 & 20.00 & 0 & 0.00 & 25 \\
\hline Ceftazidime & 12 & 48.00 & 13 & 52.00 & 0 & 0.00 & 25 \\
\hline Cipro & 100 & 69.93 & 43 & 30.07 & 0 & 0.00 & 143 \\
\hline Clinda & 70 & 52.24 & 63 & 47.01 & 1 & 0.75 & 134 \\
\hline Cotri & 83 & 58.04 & 60 & 41.96 & 0 & 0.00 & 143 \\
\hline Eryth & 90 & 62.94 & 53 & 37.06 & 0 & 0.00 & 143 \\
\hline Genta & 58 & 100.00 & 0 & 0.00 & 0 & 0.00 & 58 \\
\hline Levo & 22 & 91.67 & 1 & 4.17 & 1 & 4.17 & 24 \\
\hline Linez & 25 & 100.00 & 0 & 0.00 & 0 & 0.00 & 25 \\
\hline Piper & 18 & 72.00 & 7 & 28.00 & 0 & 0.00 & 25 \\
\hline Tetra & 7 & 87.50 & 1 & 12.50 & 0 & 0.00 & 8 \\
\hline Teico & 2 & 100.00 & 0 & 0.00 & 0 & 0.00 & 2 \\
\hline Vanco & 25 & 100.00 & 0 & 0.00 & 0 & 0.00 & 25 \\
\hline & & & & & & & \\
\hline
\end{tabular}


Fig.1 Age group Distribution of CONS infection $(n=143)$

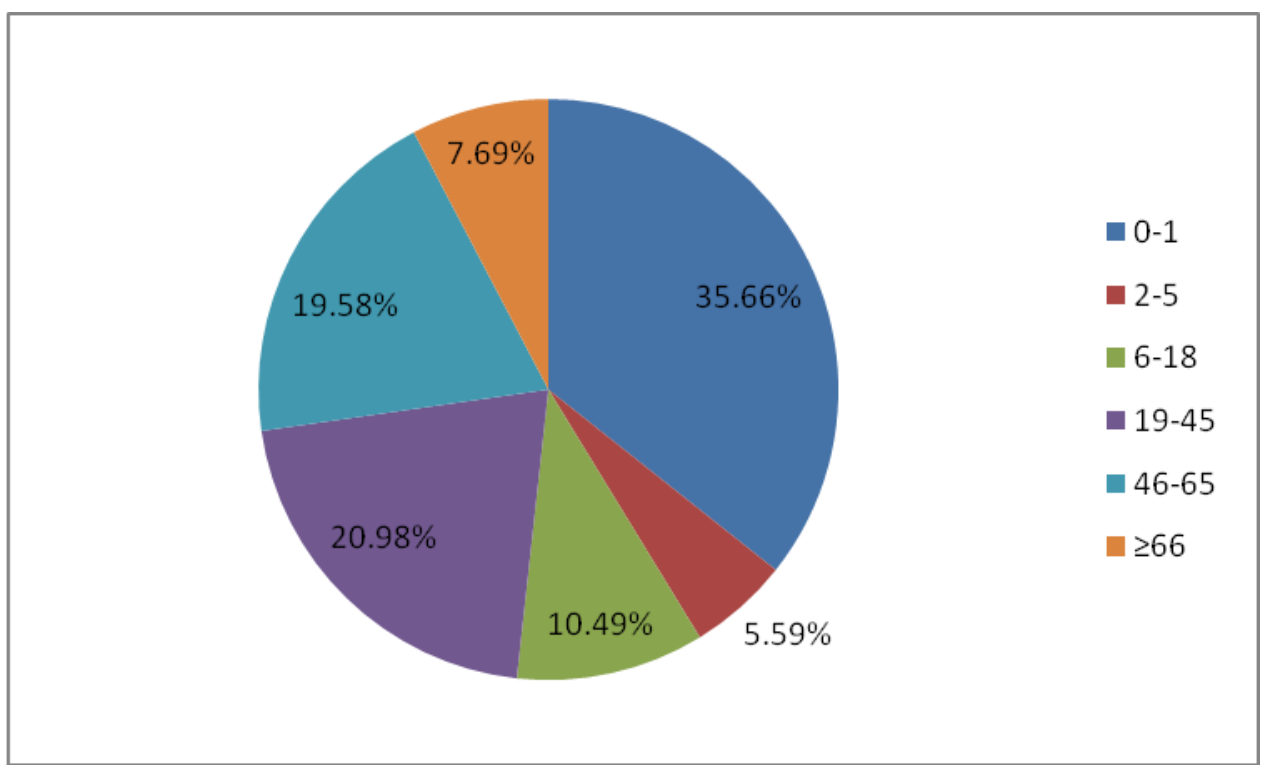

Fig.2 Sensitivity of CoNS

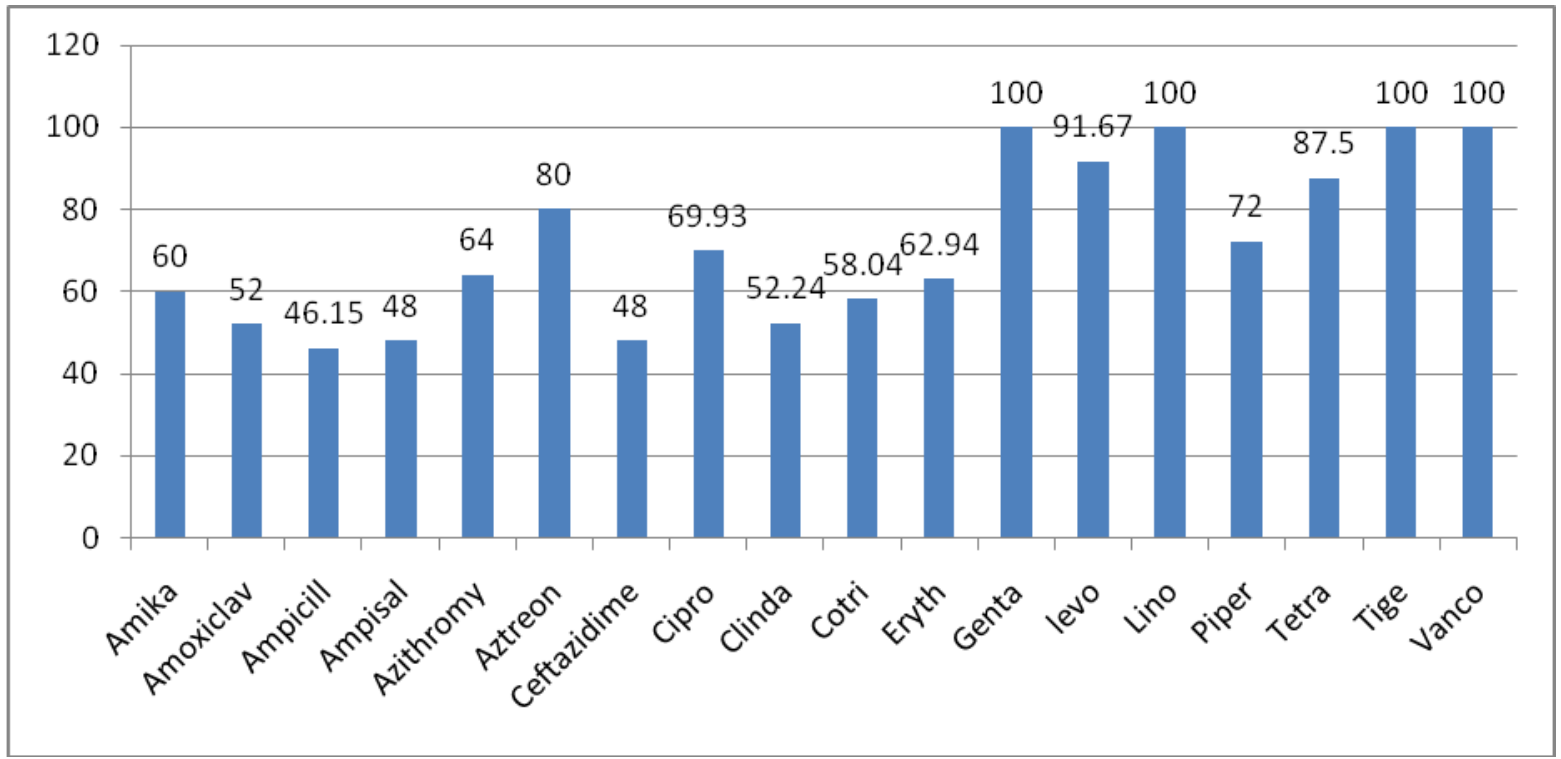


Fig.3 Resistance of CoNS

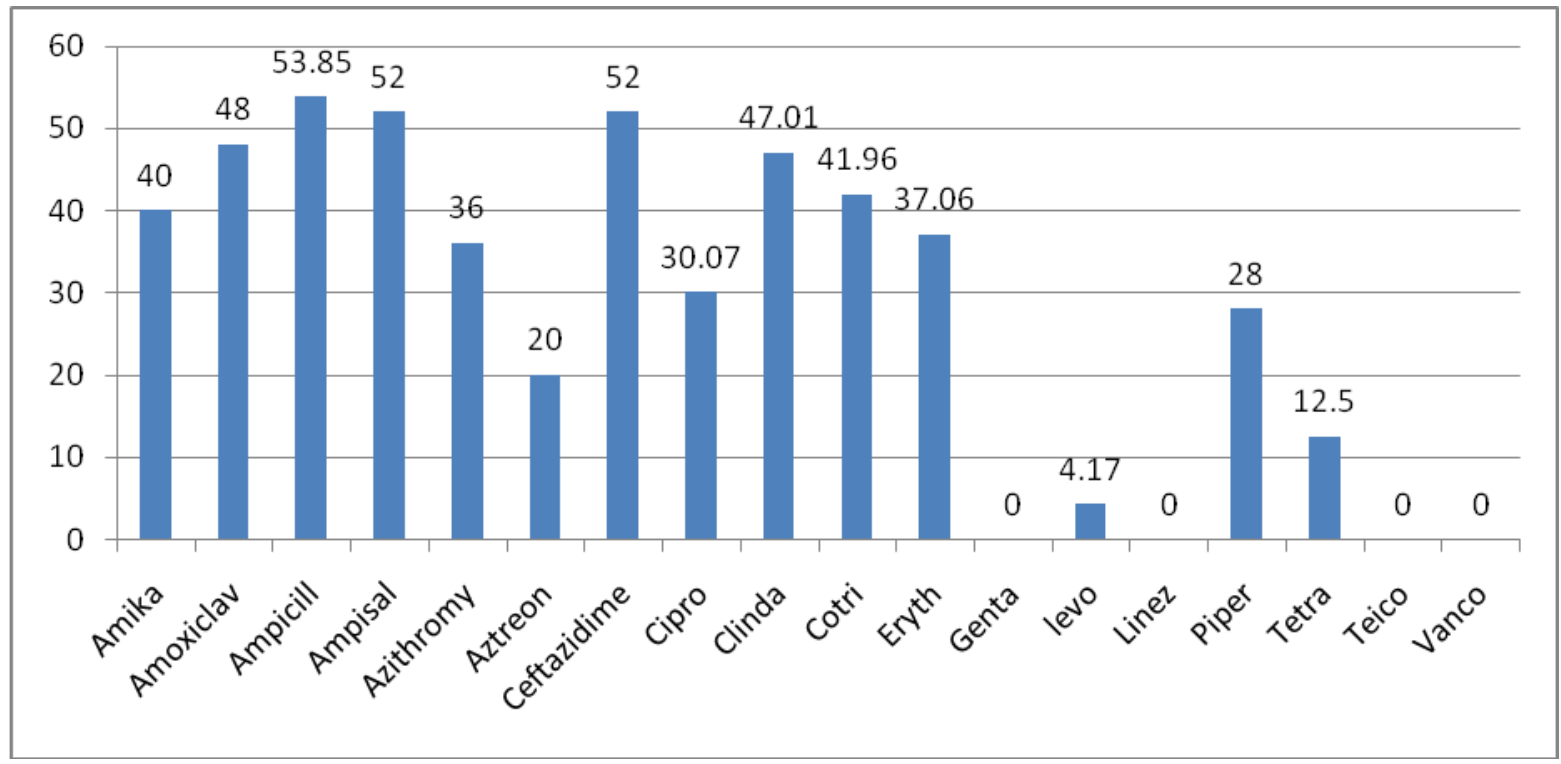

Then, based on the antibiotic susceptibility report, they start an antibiotic especially targeted to the particular bacteria. ${ }^{2,6}$ MRSA isolates can show marked geographic variation in their patterns of multi-drug resistance, most likely reflecting the properties of unique multiresistant MRSA clones dominant in the different institutions that have different antibiotic pressures. ${ }^{7}$ CoNS was one of the most common organisms among GPC isolates and the commonest isolated in the neonate and infant age group. All the CoNS isolates showed maximum sensitivity to Vancomycin, Gentamycin, Teicoplanin, and Linezolid while it showed high-level resistance to Penicillin, Ceftazidime, Clindamycin, and Cotrimoxazole. Strict aseptic procedures to be implemented during patient's stay in the hospital to minimize nosocomial infections. ${ }^{7}$

\section{References}

Rahman Z A, Hamzah S H, Hassan S H, et al., The significance of coagulase-negative staphylococci bacteremia in a low resource setting. J Infect Dev Ctries 2013; 7(6):448-452.

Healthline.

Available

at: https://www.healthline.com/health/coa gulase-negative-staph (Accessed on 15 July 2016)

Michalik, M., Samet, A., Podbielska-Kubera, A. et al., Coagulase-negative staphylococci (CoNS) as a significant etiological factor of laryngological infections: a review. Ann Clin Microbiol Antimicrob 19, 26 (2020).

Seifert $H$, Wisplinghoff $H$. Bacteriology General. In: Borriello S P, Murray R P, Funke $\mathrm{G}$ editors. Topley, and Wilsons Microbiology and Microbiological infections. 10th ed. 2010 ;(1) : 509-16.

Young L S. Sepsis syndrome. In: Mandell G L, Mandell J E, Benett J E, Dolin R editors. Principles and practice of infectious diseases, $5^{\text {th }}$ ed Churchill Living stone; New York: 2005: 80619.

Becker K, Heilmann Cand Peters G. Coagulase-Negative Staphylococci. Clin Microbiol Rev. 2014 Oct; 27(4): 870-926.

Microb Drug ResistI Santos Sanches et al., Pattern of multidrug resistance among methicillin-resistant hospital isolates of coagulase-positive and coagulase- 
negative staphylococci collected in the international multicenter study 2000;6(3):199-211.

DOI:

RESIST in 1997 and 1998 Fall

\section{How to cite this article:}

Pankaj Katoch, Ambika Sood and Surinder Singh. 2021. Coagulase-Negative Staphylococci (CoNS) Positivity and Sensitivity in Invasive Blood Stream Infections using Automated Bactec in Tertiary care Hospital of a Hilly State in India. Int.J.Curr.Microbiol.App.Sci. 10(08): 523530. doi: https://doi.org/10.20546/ijcmas.2021.1008.062 